\title{
A STATISTIC FOR TESTING THE EQUALITY OF EIGENVALUE OF COVARIANCE MATRIX ON MULTIPOPULATION
}

\author{
Hidetoshi Murakami*, Shin-ichi Tsukada ${ }^{\dagger}$ and Yuichi Takeda ${ }^{\ddagger}$
}

\begin{abstract}
A test statistic for the equality of the $j$-th largest eigenvalues of the covariance matrix in a multipopulation is proposed. Asymptotic distribution of the statistic is derived under the normal population when the sample sizes are equal. By simulation studies, we investigate the power of a test using the suggested statistic for normal, contaminated normal and skew normal populations, and compare it with two nonparametric tests.
\end{abstract}

\section{Introduction}

Principal component analysis (PCA) is an extremely important technique in multivariate analysis from both practical and theoretical viewpoints. Recent developments in computerized measurement technology have permitted the accumulation of multivariate data. The analysis of multidimensional data is particularly important in studies of DNA microarrays, finance, environmental threats, image analysis, functional data, and other similar data. There have been many books and papers concerning PCA (Anderson, 2003; Jackson, 2003; Jolliffe, 2002). However, under the non-normal population family such as contaminated normal populations, lognormal populations and skew normal populations, it is very difficult to obtain the exact distribution of eigenvalues of a covariance matrix. Therefore, there have not been many papers on testing the hypothesis that the $j$-th largest eigenvalues are equal in bipopulations and in multipopulations. Therefore we are interested in testing the equality of the $j$-th largest eigenvalues of the covariance matrix, and we treat the contaminated normal and skew normal populations as examples of non-normal populations in this paper. For bipopulations, Sugiyama and Ushizawa (1998) propose a testing procedure for the equality of the $j$-th largest eigenvalues by applying the Ansari-Bradley test (1960). In addition, Takeda (2001) proposed a test statistic and derived the exact distribution of the statistic under normality. Recently, Murakami, Hino and Tsukada (2007) extended the testing procedure, which uses two nonparametric tests, from a bipopulation to a multipopulation. However, we need large sample sizes to obtain high power when we use a nonparametric test. Therefore the purpose of this paper is to propose a more effective statistic for testing the equality of the $j$-th largest eigenvalues in multipopulation. To examine the equality of the $j$-th largest eigenvalues, we test the equality of the variance of the principal component scores.

Suppose that $\boldsymbol{x}_{1}^{(i)}, \ldots, \boldsymbol{x}_{N_{i}}^{(i)}$ are random observations from a $p$-dimensional distribution with mean $\boldsymbol{\mu}_{i}$ and covariance matrix $\boldsymbol{\Sigma}_{i}$, where $i=1, \ldots, k$. Let $\lambda_{j}^{(i)}$ be the $j$-th largest

\footnotetext{
*Department of Industrial and Systems Engineering, Chuo University, 1-13-27 Kasuga, Bunkyo-ku, Tokyo 112-8551, Japan E-mail: murakami@indsys.chuo-u.ac.jp

†School of Science and Engineering, Meisei University, 2-1-1 Hodokubo, Hino-shi, Tokyo, 191-8506, Japan

${ }_{\ddagger}$ Kanagawa Institute of Technology, 1030 Shimo-Ogino, Atsugi-shi, Kanagawa 243-0292, Japan

Key words: Eigenvalues, Principal component score, Power comparison
} 
eigenvalue of the covariance matrix $\boldsymbol{\Sigma}_{i}$ in the $i$-th population. For fixed $j$, we consider testing the hypothesis

$$
H_{0}: \lambda_{j}^{(1)}=\lambda_{j}^{(2)}=\cdots=\lambda_{j}^{(k)} \quad \text { against } \quad H_{1}: \operatorname{not} H_{0} .
$$

Let $\boldsymbol{h}_{j}^{(i)}$ be the sample eigenvector corresponding to the $j$-th largest sample eigenvalue $l_{j}^{(i)}$ of the $i$-th sample covariance matrix given by

$$
\boldsymbol{S}^{(i)}=\frac{1}{N_{i}-1} \sum_{\alpha=1}^{N_{i}}\left(\boldsymbol{x}_{\alpha}^{(i)}-\overline{\boldsymbol{x}}^{(i)}\right)\left(\boldsymbol{x}_{\alpha}^{(i)}-\overline{\boldsymbol{x}}^{(i)}\right)^{\prime},
$$

where $\overline{\boldsymbol{x}}^{(i)}$ is the sample mean vector of the $i$-th population. We express the $j$-th principal component score as

$$
y_{j \alpha}^{(i)}=\boldsymbol{h}_{j}^{(i) \prime}\left(\boldsymbol{x}_{\alpha}^{(i)}-\overline{\boldsymbol{x}}^{(i)}\right), \quad \alpha=1, \ldots, N_{i} .
$$

In Section 2, we develop a testing procedure in multipopulation and propose a new test statistic, namely $T_{j}$. We also derive the asymptotic distribution of the statistic $T_{j}$ under a normal population when sample sizes are equal. In Section 3 , we simulate to investigate the type I error and the power of a test using the statistic $T_{j}$ under various populations and to compare the power of the test with two nonparametric tests. In this paper, we treat the normal population, the contaminated normal population as a symmetric non-normal population and the skew normal population as an asymmetric non-normal population. Finally, concluding remarks are given in Section 4.

\section{Test statistic and asymptotic distribution}

In this section, we suggest a new test statistic and derive its asymptotic distribution. To test the equality of the $j$-th largest eigenvalues in the $k$-population, we use the principal component scores $Y_{i}$ as

$$
Y_{1}=\left\{y_{j 1}^{(1)}, y_{j 2}^{(1)}, \ldots, y_{j N_{1}}^{(1)}\right\}, Y_{2}=\left\{y_{j 1}^{(2)}, y_{j 2}^{(2)}, \ldots, y_{j N_{2}}^{(2)}\right\}, \cdots, Y_{k}=\left\{y_{j 1}^{(k)}, y_{j 2}^{(k)}, \ldots, y_{j N_{k}}^{(k)}\right\}
$$

Under normal populations, the eigenvalues of the covariance matrices are equal to the variances of the principal component scores. For non-normal populations, Murakami et al. (2007) give the variance of the principal component score $y_{j \alpha}^{(i)}$ as follows:

$$
\operatorname{Var}\left[y_{j \alpha}^{(i)}\right]=\lambda_{j}^{(i)}-\frac{2}{N_{i}-1} \sum_{q \neq j}^{p} \frac{\mathrm{E}\left[x_{q \alpha}^{(i) 2} x_{j \alpha}^{(i) 2}\right]}{\lambda_{q}^{(i)}-\lambda_{j}^{(i)}}+O\left(N_{i}^{-2}\right),
$$

where $x_{j \alpha}^{(i)}$ is the $j$-th element of $\boldsymbol{x}_{\alpha}^{(i)}$. From equation (1), we may treat testing the null hypothesis as testing the equality of variances of principal component scores when the sample sizes $N_{i}$ are sufficiently large. When the differences between the $j$-th largest eigenvalue and other eigenvalues in each population are sufficiently large, the term of $O\left(N_{i}^{-1}\right)$ may not affect the test of equality of the $j$-th largest eigenvalues.

Now we propose a statistic $T_{j}$ for testing the hypothesis as follows:

$$
T_{j}=\frac{N}{2 k} \sum_{1 \leq \omega<\beta \leq k} \sum_{1}\left(\log \frac{l_{j}^{(\beta)}}{l_{j}^{(\omega)}}\right)^{2}
$$


where

$$
N=\sum_{i=1}^{k} N_{i}, \quad l_{j}^{(\omega)}=\frac{1}{N_{\omega}} \sum_{i=1}^{N_{\omega}}\left(y_{j i}^{(\omega)}-\bar{y}_{j}^{(\omega)}\right)^{2} \quad \text { and } \quad \bar{y}_{j}^{(\omega)}=\frac{1}{N_{\omega}} \sum_{i=1}^{N_{\omega}} y_{j i}^{(\omega)} .
$$

Assuming normal populations, we derive the limiting distribution of the statistic $T_{j}$ as the following theorem.

Theorem 1 When $N_{1}=N_{2}=\cdots=N_{k} \equiv n+1$, the limiting distribution of the statistic $T_{j}$ is the $\chi^{2}$ distribution with $(k-1)$ degrees of freedom under normal populations.

Proof. The limiting distribution of

$$
z_{j}=\sqrt{n}\left(\begin{array}{c}
\log l_{j}^{(1)}-\log \lambda_{j}^{(1)} \\
\log l_{j}^{(2)}-\log \lambda_{j}^{(2)} \\
\vdots \\
\log l_{j}^{(k)}-\log \lambda_{j}^{(k)}
\end{array}\right)
$$

is $N\left(\mathbf{0}, 2 I_{k}\right)$ when the population is normal (Anderson, 2003). Under the null hypothesis,

$$
A \boldsymbol{z}_{j}=\sqrt{n}\left(\begin{array}{c}
\log l_{j}^{(1)}-\log l_{j}^{(2)} \\
\log l_{j}^{(2)}-\log l_{j}^{(3)} \\
\vdots \\
\log l_{j}^{(k-1)}-\log l_{j}^{(k)}
\end{array}\right)
$$

is asymptotically distributed as $N\left(\mathbf{0}, 2 A A^{\prime}\right)$, where

$$
A=\left(\begin{array}{ccccc}
1 & -1 & 0 & & \\
0 & 1 & -1 & 0 & \\
& \mathbf{0} & \ddots & \ddots & \\
& & & 1 & -1
\end{array}\right)
$$

Therefore, we may obtain that

$$
\left(A z_{j}\right)^{\prime}\left(2 A A^{\prime}\right)^{-1}\left(A z_{j}\right)=\frac{1}{2} z_{j}^{\prime}\left\{A^{\prime}\left(A A^{\prime}\right)^{-1} A\right\} z_{j}
$$

is asymptotically distributed as a $\chi^{2}$ distribution with $(k-1)$ degrees of freedom. Noting that

$$
k A^{\prime}\left(A A^{\prime}\right)^{-1} A=\left(\begin{array}{cccc}
k-1 & -1 & \cdots & -1 \\
-1 & k-1 & \cdots & -1 \\
\vdots & \vdots & \ddots & \vdots \\
-1 & -1 & \cdots & k-1
\end{array}\right)
$$

we have

$$
k \boldsymbol{z}_{j}^{\prime}\left\{A^{\prime}\left(A A^{\prime}\right)^{-1} A\right\} \boldsymbol{z}_{j}=n \sum_{1 \leq \omega<\beta \leq k} \sum_{1}\left(\log l_{j}^{(\beta)}-\log l_{j}^{(\omega)}\right)^{2}
$$


Therefore the limiting distribution of

$$
\frac{1}{2} z_{j}^{\prime}\left\{A^{\prime}\left(A A^{\prime}\right)^{-1} A\right\} z_{j}=\frac{n}{2 k} \sum_{1 \leq \omega<\beta \leq k} \sum_{1}\left(\log l_{j}^{(\beta)}-\log l_{j}^{(\omega)}\right)^{2}=T_{j}
$$

is a $\chi^{2}$ distribution with $(k-1)$ degrees of freedom.

For the case of unequal sample sizes and non-normal populations, it is difficult to derive the limiting distribution of the statistic $T_{j}$ because the weight is different for each $\log l_{j}^{(\beta)}-\log l_{j}^{(\omega)}$. Then the limiting distribution of the statistic $T_{j}$ may not be a simple expression such as a $\chi^{2}$ distribution with $(k-1)$ degrees of freedom. Therefore, we adopt a permutation test for the statistic $T_{j}$ to examine the equality of eigenvalues in next section.

\section{Simulation study}

In this section, we investigate the type I error and the power of tests for the equality of the $j$-th largest eigenvalues at a significance level of $5 \%$. We carry out simulations for normal populations, contaminated normal populations as an example of symmetric nonnormal populations and skew normal populations as an example of asymmetric non-normal populations. Let the number of populations be three. The simulations are repeated 100,000 times with 10,000 permutations for the statistic $T_{j}$. Therefore, the type I error and the power maintain precision down to two decimal places.

Now we give the generalized Ansari-Bradley statistic. The Ansari-Bradley test is known as a method of testing the variances. One of the assumptions for the Ansari-Bradley test is that the samples are independent. Sugiyama and Ushizawa (1998) prove that the degree of dependence between each principal component score is weak when the sample size is sufficiently large under normality. They show the Ansari-Bradley test could be applicable to test the equality of the eigenvalues using principal component scores. Let $R_{j m}^{(i)}$ be the increasing order rank of $y_{j m}^{(i)}$ in the combined $N$ observations. The Ansari-Bradley statistic for $k$-sample is given by

$$
A B_{j k}= \begin{cases}\frac{48(N-1)}{N\left(N^{2}-4\right)} \sum_{i=1}^{k} N_{i}\left(\bar{A}_{j}{ }^{(i)}-\frac{N+2}{4}\right)^{2} & (N \text { is even }) \\ \frac{48 N^{2}}{N(N+1)\left(N^{2}+3\right)} \sum_{i=1}^{k} N_{i}\left(\bar{A}_{j}{ }^{(i)}-\frac{(N+1)^{2}}{4 N}\right)^{2}(N \text { is odd }),\end{cases}
$$

where

$$
{\overline{A_{j}}}^{(i)}=\frac{1}{N_{i}} \sum_{m=1}^{N_{i}}\left(\frac{N+1}{2}-\left|R_{j m}^{(i)}-\frac{N+1}{2}\right|\right) .
$$

The limiting distribution of the Ansari-Bradley statistic for a $k$-population is a $\chi^{2}$ distribution with $(k-1)$ degrees of freedom under the null hypothesis (Tsai et al., 1975). Gibbons and Chakraborti (2003) indicate that the asymptotic relative efficiency of the test by Mood (1954), the Mood test, is higher than that of the Ansari-Bradley test. The Mood statistic for a $k$-sample is known as

$$
M_{j k}=\frac{180}{N(N+1)\left(N^{2}-4\right)} \sum_{i=1}^{k} N_{i}\left(\bar{M}_{j}^{(i)}-\frac{N^{2}-1}{12}\right)^{2},
$$


where

$$
\bar{M}_{j}{ }^{(i)}=\frac{1}{N_{i}} \sum_{m=1}^{N_{i}}\left(R_{j m}^{(i)}-\frac{N+1}{2}\right)^{2} .
$$

The limiting distribution of the Mood statistic $M_{j k}$ is also a $\chi^{2}$ distribution with $(k-1)$ degrees of freedom (Tsai et al., 1975). Therefore we set the critical value of both test as 5.991 for $k=3$.

Murakami et al. (2007) show how the statistic $A B_{j k}$ and the statistic $M_{j k}$ are used to test the hypothesis. We simulate 100,000 times for the statistics $A B_{j k}$ and $M_{j k}$, and investigate the type I error and the power of the test using the statistics $T_{j}, A B_{j k}$ and $M_{j k}$. We set the populations as normal populations $N_{3}\left(\mathbf{0}, \boldsymbol{\Sigma}^{(i)}\right)$ and contaminated normal populations $0.95 \times N_{3}\left(\mathbf{0}, \boldsymbol{\Sigma}^{(i)}\right)+0.05 \times N_{3}\left(\mathbf{0}, 3 \boldsymbol{\Sigma}^{(i)}\right)$, and the sample sizes are $N_{1}=N_{2}=$ $N_{3}=50, N_{1}=N_{2}=N_{3}=100$ and $N_{1}=200, N_{2}=150, N_{3}=100$. We treat the same cases as Murakami et al. (2007) and add the case of $N_{1}=150, N_{2}=100, N_{3}=50$ to compare the type I error and the power for the case of $N_{1}=N_{2}=N_{3}=100$. Since the distribution of the eigenvalues may not depend on the eigenvector, it is sufficient to examine only the case that the population covariance matrices are diagonal without loss of generality. We set the population covariance matrices as

$$
\boldsymbol{\Sigma}^{(i)}=\operatorname{diag}\left(\lambda_{1}^{(i)}, \lambda_{2}^{(i)}, \lambda_{3}^{(i)}\right)
$$

and treat the following four cases.

Case 1:

$\lambda_{1}^{(1)}=6, \lambda_{2}^{(1)}=3, \lambda_{3}^{(1)}=1 ; \quad \lambda_{1}^{(2)}=6, \lambda_{2}^{(2)}=3, \lambda_{3}^{(2)}=1 ; \quad \lambda_{1}^{(3)}=6, \lambda_{2}^{(3)}=3, \lambda_{3}^{(3)}=1$,

Case 2:

$\lambda_{1}^{(1)}=10, \lambda_{2}^{(1)}=3, \lambda_{3}^{(1)}=1 ; \quad \lambda_{1}^{(2)}=8, \lambda_{2}^{(2)}=3, \lambda_{3}^{(2)}=1 ; \quad \lambda_{1}^{(3)}=6, \lambda_{2}^{(3)}=3, \lambda_{3}^{(3)}=1$,

Case 3:

$\lambda_{1}^{(1)}=9, \lambda_{2}^{(1)}=5, \lambda_{3}^{(1)}=2 ; \quad \lambda_{1}^{(2)}=6, \lambda_{2}^{(2)}=3, \lambda_{3}^{(2)}=1 ; \quad \lambda_{1}^{(3)}=6, \lambda_{2}^{(3)}=3, \lambda_{3}^{(3)}=1$,

Case 4:

$\lambda_{1}^{(1)}=9, \lambda_{2}^{(1)}=5, \lambda_{3}^{(1)}=2 ; \lambda_{1}^{(2)}=7.5, \lambda_{2}^{(2)}=4, \lambda_{3}^{(2)}=1.5 ; \lambda_{1}^{(3)}=6, \lambda_{2}^{(3)}=3, \lambda_{3}^{(3)}=1$.

Case 1 is under the null hypothesis for all eigenvalues. Case 2 is that only the largest eigenvalues are different and other eigenvalues are under the null hypothesis. Case 3 and Case 4 is that all eigenvalues are different. In the all of Tables, the results on the left of the dividing lines shows the type I error of the statistics. In addition, the results on the right of the dividing lines reveal the power of tests using the $M_{j k}, A B_{j k}$ and $T_{j}$ statistics. Table 1 and Table 2 represent the results for the normal population. 
Table 1: Normal populations

\begin{tabular}{|cc||cccc||cc|ccc|}
\hline \multicolumn{1}{|c||}{} & \multicolumn{4}{c||}{$N_{1}=N_{2}=N_{3}=50$} & \multicolumn{4}{c|}{$N_{1}=N_{2}=N_{3}=100$} \\
\cline { 3 - 10 } & & Case 1 & Case 2 & Case 3 & Case 4 & Case 1 & Case 2 & Case 3 & Case 4 \\
\hline \hline$j=1$ & $M_{1 k}$ & 0.041 & 0.242 & 0.224 & 0.169 & 0.046 & 0.478 & 0.428 & 0.327 \\
& $A B_{1 k}$ & 0.042 & 0.201 & 0.184 & 0.144 & 0.047 & 0.395 & 0.348 & 0.269 \\
& $T_{1}$ & $\mathbf{0 . 0 4 0}$ & $\mathbf{0 . 3 0 2}$ & $\mathbf{0 . 2 6 9}$ & $\mathbf{0 . 2 0 6}$ & $\mathbf{0 . 0 4 5}$ & $\mathbf{0 . 5 9 3}$ & $\mathbf{0 . 5 3 1}$ & $\mathbf{0 . 4 1 5}$ \\
$j=2$ & $M_{2 k}$ & 0.048 & 0.049 & 0.335 & 0.253 & 0.049 & 0.050 & 0.615 & 0.488 \\
& $A B_{2 k}$ & 0.048 & 0.049 & 0.271 & 0.211 & 0.049 & 0.050 & 0.511 & 0.403 \\
& $T_{2}$ & $\mathbf{0 . 0 4 7}$ & $\mathbf{0 . 0 4 9}$ & $\mathbf{0 . 4 0 8}$ & $\mathbf{0 . 3 1 5}$ & $\mathbf{0 . 0 4 8}$ & $\mathbf{0 . 0 5 0}$ & $\mathbf{0 . 7 3 3}$ & $\mathbf{0 . 6 0 1}$ \\
$j=3$ & $M_{3 k}$ & 0.056 & 0.056 & 0.564 & 0.445 & 0.053 & 0.053 & 0.867 & 0.763 \\
& $A B_{3 k}$ & 0.055 & 0.055 & 0.465 & 0.370 & 0.052 & 0.052 & 0.776 & 0.664 \\
& $T_{3}$ & $\mathbf{0 . 0 5 7}$ & $\mathbf{0 . 0 5 6}$ & $\mathbf{0 . 6 6 6}$ & $\mathbf{0 . 5 5 0}$ & $\mathbf{0 . 0 5 4}$ & $\mathbf{0 . 0 5 4}$ & $\mathbf{0 . 9 4 2}$ & $\mathbf{0 . 8 7 1}$ \\
\hline
\end{tabular}

Table 2: Normal populations

\begin{tabular}{|cc||cccc||cc|ccc|}
\hline \multicolumn{1}{|c||}{} & \multicolumn{9}{c||}{$N_{1}=150, N_{2}=100, N_{3}=50$} & \multicolumn{3}{c|}{$N_{1}=200, N_{2}=150, N_{3}=100$} \\
\cline { 3 - 9 } & & Case 1 & Case 2 & Case 3 & Case 4 & Case 1 & Case 2 & Case 3 & Case 4 \\
\hline \hline$j=1$ & $M_{1 k}$ & 0.045 & 0.367 & 0.453 & 0.241 & 0.047 & 0.613 & 0.641 & 0.420 \\
& $A B_{1 k}$ & 0.046 & 0.309 & 0.375 & 0.207 & 0.048 & 0.518 & 0.538 & 0.349 \\
& $T_{1}$ & $\mathbf{0 . 0 4 3}$ & $\mathbf{0 . 4 5 8}$ & $\mathbf{0 . 4 0 8}$ & $\mathbf{0 . 3 0 7}$ & $\mathbf{0 . 0 4 6}$ & $\mathbf{0 . 7 3 7}$ & $\mathbf{0 . 6 8 7}$ & $\mathbf{0 . 5 2 9}$ \\
$j=2$ & $M_{2 k}$ & 0.049 & 0.050 & 0.695 & 0.428 & 0.049 & 0.050 & 0.852 & 0.641 \\
& $A B_{2 k}$ & 0.049 & 0.050 & 0.594 & 0.361 & 0.049 & 0.050 & 0.761 & 0.545 \\
& $T_{2}$ & $\mathbf{0 . 0 4 9}$ & $\mathbf{0 . 0 5 0}$ & $\mathbf{0 . 6 8 0}$ & $\mathbf{0 . 5 3 4}$ & $\mathbf{0 . 0 4 9}$ & $\mathbf{0 . 0 5 0}$ & $\mathbf{0 . 8 9 8}$ & $\mathbf{0 . 7 6 8}$ \\
$j=3$ & $M_{3 k}$ & 0.055 & 0.055 & 0.928 & 0.703 & 0.052 & 0.052 & 0.985 & 0.898 \\
& $A B_{3 k}$ & 0.055 & 0.055 & 0.861 & 0.613 & 0.051 & 0.052 & 0.956 & 0.823 \\
& $T_{3}$ & $\mathbf{0 . 0 6 0}$ & $\mathbf{0 . 0 6 0}$ & $\mathbf{0 . 9 3 3}$ & $\mathbf{0 . 8 1 5}$ & $\mathbf{0 . 0 5 5}$ & $\mathbf{0 . 0 5 5}$ & $\mathbf{0 . 9 9 4}$ & $\mathbf{0 . 9 6 2}$ \\
\hline
\end{tabular}

The simulation results show the validity of the permutation test and nonparametric tests. Every test may be conservative for $j=1,2$ under the null hypothesis. The nonparametric tests are more robust than the test using the statistic $T_{j}$. However, the power of the test using the statistic $T_{j}$ is the greatest in both cases regardless of whether the sample sizes are equal or unequal, except only for Case 3 in Table 2. The power of Mood's test is greatest for $j=1$ and 2 for Case 3 in the left of Table 2. The total sample sizes are equal in the right of Table 1 and the left of Table 2 . The power of the test using the statistic $T_{j}$ tends to decrease when the sample sizes are unequal. Further when we apply the permutation test for the statistic $M_{j k}$ and $A B_{j k}$, their powers are almost equivalent to the power of the Mood test and Ansari-Bradley test.

In the study of environmental problems, the contaminated normal distribution is used a lot. Therefore we treat the contaminated normal population as a symmetric non-normal population in Table 3 and Table 4 . Table 3 indicates the results for the cases of equal sample sizes. We show the results of tests for unequal sample sizes in Table 4 . 
Testing the Equality of Eigenvalues

Table 3: Contaminated normal populations

\begin{tabular}{|c|c|c|c|c|c|c|c|c|c|}
\hline & \multicolumn{4}{|c|}{$N_{1}=N_{2}=N_{3}=50$} & \multicolumn{4}{|c|}{$N_{1}=N_{2}=N_{3}=100$} \\
\hline & & Case 1 & Case 2 & Case 3 & Case 4 & Case 1 & Case 2 & Case 3 & Case 4 \\
\hline \multirow[t]{3}{*}{$j=1$} & $M_{1 k}$ & 0.040 & 0.233 & 0.215 & 0.163 & 0.044 & $\overline{0.462}$ & 0.411 & 0.311 \\
\hline & $A B_{1 k}$ & 0.042 & 0.198 & 0.178 & 0.141 & 0.045 & 0.386 & 0.337 & 0.260 \\
\hline & $T_{1}$ & 0.033 & 0.261 & 0.228 & 0.174 & 0.037 & 0.512 & 0.452 & 0.344 \\
\hline \multirow[t]{3}{*}{$j=2$} & $M_{2 k}$ & 0.048 & 0.048 & 0.329 & 0.248 & 0.047 & 0.048 & 0.601 & 0.473 \\
\hline & $A B_{2 k}$ & 0.048 & 0.048 & 0.268 & 0.208 & 0.048 & 0.048 & 0.501 & 0.395 \\
\hline & $T_{2}$ & 0.046 & 0.047 & 0.379 & 0.293 & 0.043 & 0.044 & 0.672 & 0.541 \\
\hline \multirow[t]{3}{*}{$j=3$} & $M_{3 k}$ & 0.055 & 0.055 & 0.555 & 0.438 & 0.051 & 0.050 & 0.858 & 0.751 \\
\hline & $A B_{3 k}$ & 0.054 & 0.054 & 0.460 & 0.366 & 0.051 & 0.050 & 0.769 & 0.655 \\
\hline & $T_{3}$ & 0.056 & 0.058 & 0.639 & 0.524 & 0.049 & 0.050 & 0.913 & 0.824 \\
\hline
\end{tabular}

Table 4: Contaminated normal populations

\begin{tabular}{|cc||cccc||cc|ccc|}
\hline \multicolumn{1}{|c||}{} & \multicolumn{3}{c||}{$N_{1}=150, N_{2}=100, N_{3}=50$} & \multicolumn{3}{c|}{$N_{1}=200, N_{2}=150, N_{3}=100$} \\
\cline { 3 - 10 } & & \multicolumn{1}{c|}{ Case 1 } & Case 2 & Case 3 & Case 4 & Case 1 & Case 2 & Case 3 & Case 4 \\
\hline \hline$j=1$ & $M_{1 k}$ & 0.042 & 0.373 & 0.452 & 0.247 & 0.045 & 0.593 & 0.617 & 0.401 \\
& $A B_{1 k}$ & 0.044 & 0.318 & 0.377 & 0.214 & 0.046 & 0.505 & 0.521 & 0.338 \\
& $T_{1}$ & $\mathbf{0 . 0 3 4}$ & $\mathbf{0 . 4 2 5}$ & $\mathbf{0 . 3 5 5}$ & $\mathbf{0 . 2 7 9}$ & $\mathbf{0 . 0 3 8}$ & $\mathbf{0 . 6 5 3}$ & $\mathbf{0 . 5 8 7}$ & $\mathbf{0 . 4 4 7}$ \\
$j=2$ & $M_{2 k}$ & 0.048 & 0.049 & 0.691 & 0.431 & 0.047 & 0.047 & 0.836 & 0.621 \\
& $A B_{2 k}$ & 0.050 & 0.050 & 0.593 & 0.365 & 0.048 & 0.048 & 0.745 & 0.531 \\
& $T_{2}$ & $\mathbf{0 . 0 4 6}$ & $\mathbf{0 . 0 4 7}$ & $\mathbf{0 . 6 3 8}$ & $\mathbf{0 . 5 1 4}$ & $\mathbf{0 . 0 4 5}$ & $\mathbf{0 . 0 4 3}$ & $\mathbf{0 . 8 4 1}$ & $\mathbf{0 . 7 0 0}$ \\
$j=3$ & $M_{3 k}$ & 0.055 & 0.055 & 0.926 & 0.705 & 0.050 & 0.050 & 0.982 & 0.887 \\
& $A B_{3 k}$ & 0.055 & 0.055 & 0.860 & 0.616 & 0.050 & 0.050 & 0.951 & 0.812 \\
& $T_{3}$ & $\mathbf{0 . 0 5 8}$ & $\mathbf{0 . 0 5 8}$ & $\mathbf{0 . 9 1 1}$ & $\mathbf{0 . 7 9 8}$ & $\mathbf{0 . 0 4 9}$ & $\mathbf{0 . 0 4 9}$ & $\mathbf{0 . 9 8 6}$ & $\mathbf{0 . 9 3 5}$ \\
\hline
\end{tabular}

The simulation results permit that we may use the $T_{j}$ statistic for testing the equality of the $j$-th largest eigenvalues for the contaminated normal population. All tests may be conservative for $j=1,2$ under the null hypothesis. If any $N_{i}$ is greater than 100 , all tests are also conservative for $j=3$. In addition, the nonparametric tests are more robust than the test using the statistic $T_{j}$, especially $j=1$. But the power of the test using the statistic $T_{j}$ is the greatest in these cases regardless of whether the sample sizes are equal. For Case 3 on unequal sample sizes, the Mood test is more suitable than the test using the statistic $T_{j}$. However, the power of the test using the statistic $T_{j}$ is higher than that of the nonparametric tests for Case 2 and Case 4 . We further applied the permutation test for the $M_{j k}$ and $A B_{j k}$ statistics. Their powers are almost equivalent to the power of the original tests. The total sample sizes are equal in the right of Table 3 and the left of Table 4, but the power of the test using the statistic $T_{j}$ tends to decrease when the sample sizes are unequal.

Finally, we simulate the skew normal populations $M S N\left(\boldsymbol{\nu}^{(i)}, \mathbf{0}, \boldsymbol{\Sigma}^{(i)}\right)$ for an asymmetric non-normal population, where $\boldsymbol{\nu}^{(i)}=\left(\nu_{1}, \nu_{2}, \nu_{3}\right)^{\prime}$ denotes the shape parameter (Genton, 2004, Chap. 1, Sec. 5). We set $\nu_{1}=\nu_{2}=\nu_{3}=0.5$. The reason why we treat this population is that we sometimes assume this population in financial markets, property markets, labor markets etc.

Table 5 to Table 8 represents the results for the skew normal populations. In Table 5 and Table 6 , we give the type I error and the power for the case of equal sample sizes. For 
the case of the unequal sample sizes, we show the type I error and the power in Table 7 and Table 8 . Let the correlation matrix be

$$
\left(\begin{array}{ccc}
1 & r_{1} & r_{2} \\
r_{1} & 1 & r_{3} \\
r_{2} & r_{3} & 1
\end{array}\right)
$$

In the case of $r=r_{1}=r_{2}=r_{3}$, the results are listed in the left of Table 5 - Table 8. When $r_{1} \neq r_{2} \neq r_{3}$, we show the results in the right of Table 5 - Table 8 .

Table 5: Skew normal populations $\left(N_{1}=N_{2}=N_{3}=50\right)$

\begin{tabular}{|cc||cccc||cccc|}
\hline \multicolumn{1}{|c||}{} & \multicolumn{5}{c|}{$r=0.5$} & \multicolumn{3}{c|}{$r_{1}=0.5, r_{2}=0.3, r_{3}=0.2$} \\
\cline { 3 - 10 } & & Case 1 & Case 2 & Case 3 & Case 4 & Case 1 & Case 2 & Case 3 & Case 4 \\
\hline \hline$j=1$ & $M_{1 k}$ & 0.049 & 0.180 & 0.270 & 0.207 & 0.049 & 0.190 & 0.258 & 0.198 \\
& $A B_{1 k}$ & 0.049 & 0.155 & 0.226 & 0.174 & 0.049 & 0.161 & 0.211 & 0.167 \\
& $T_{1}$ & $\mathbf{0 . 0 4 9}$ & $\mathbf{0 . 2 2 0}$ & $\mathbf{0 . 3 2 6}$ & $\mathbf{0 . 2 5 4}$ & $\mathbf{0 . 0 4 9}$ & $\mathbf{0 . 2 3 2}$ & $\mathbf{0 . 3 1 2}$ & $\mathbf{0 . 2 4 3}$ \\
$j=2$ & $M_{2 k}$ & 0.050 & 0.057 & 0.319 & 0.243 & 0.045 & 0.051 & 0.321 & 0.241 \\
& $A B_{2 k}$ & 0.050 & 0.055 & 0.259 & 0.203 & 0.046 & 0.050 & 0.261 & 0.201 \\
& $T_{2}$ & $\mathbf{0 . 0 5 1}$ & $\mathbf{0 . 0 6 0}$ & $\mathbf{0 . 3 8 9}$ & $\mathbf{0 . 3 0 2}$ & $\mathbf{0 . 0 4 5}$ & $\mathbf{0 . 0 5 1}$ & $\mathbf{0 . 3 9 1}$ & $\mathbf{0 . 2 9 9}$ \\
$j=3$ & $M_{3 k}$ & 0.056 & 0.056 & 0.545 & 0.429 & 0.054 & 0.054 & 0.548 & 0.428 \\
& $A B_{3 k}$ & 0.055 & 0.055 & 0.449 & 0.356 & 0.053 & 0.053 & 0.450 & 0.356 \\
& $T_{3}$ & $\mathbf{0 . 0 5 8}$ & $\mathbf{0 . 0 5 8}$ & $\mathbf{0 . 6 4 5}$ & $\mathbf{0 . 5 2 9}$ & $\mathbf{0 . 0 5 4}$ & $\mathbf{0 . 0 5 5}$ & $\mathbf{0 . 6 5 0}$ & $\mathbf{0 . 5 3 0}$ \\
\hline
\end{tabular}

Table 6: Skew normal populations $\left(N_{1}=N_{2}=N_{3}=100\right)$

\begin{tabular}{|c|c|c|c|c|c|c|c|c|c|}
\hline & \multicolumn{4}{|c|}{$r=0.5$} & \multicolumn{4}{|c|}{$r_{1}=0.5, r_{2}=0.3, r_{3}=0.2$} \\
\hline & & Case 1 & Case 2 & Case 3 & Case 4 & Case 1 & Case 2 & Case 3 & Case 4 \\
\hline \multirow[t]{3}{*}{$\overline{j=1}$} & $M_{1 k}$ & 0.050 & 0.336 & 0.500 & $\overline{0.3}$ & 0.050 & 0.356 & 0.479 & 0.371 \\
\hline & $A B_{1 k}$ & 0.050 & 0.277 & 0.409 & 0.319 & 0.050 & 0.293 & 0.391 & 0.305 \\
\hline & $T_{1}$ & 0.050 & 0.423 & 0.609 & 0.486 & 0.049 & 0.448 & 0.586 & 0.466 \\
\hline \multirow[t]{3}{*}{$j=2$} & $M_{2 k}$ & 0.050 & 0.063 & 0.578 & 0.456 & 0.048 & 0.058 & 0.585 & 0.459 \\
\hline & $A B_{2 k}$ & 0.050 & 0.061 & 0.478 & 0.376 & 0.049 & 0.056 & 0.482 & 0.379 \\
\hline & $T_{2}$ & 0.050 & 0.066 & 0.695 & 0.566 & 0.048 & 0.059 & 0.703 & 0.570 \\
\hline \multirow[t]{3}{*}{$j=3$} & $M_{3 k}$ & 0.053 & 0.053 & 0.851 & 0.743 & 0.052 & 0.053 & 0.859 & 0.753 \\
\hline & $A B_{3 k}$ & 0.052 & 0.053 & 0.757 & 0.643 & 0.052 & 0.052 & 0.766 & 0.653 \\
\hline & $T_{3}$ & 0.054 & 0.054 & 0.930 & 0.851 & 0.053 & 0.053 & 0.936 & 0.861 \\
\hline
\end{tabular}

For equal sample sizes, the simulation results reveal that all tests maintain a significant level for Case 1. The nonparametric tests are more robust than the test using the statistic $T_{j}$. However, the power of the test using the statistic $T_{j}$ is higher than the power of the Ansari-Bradley test or Mood test under the alternative hypothesis. Next we show the results for unequal sample sizes. 
Testing the Equality of Eigenvalues

Table 7: Skew normal populations $\left(N_{1}=150, N_{2}=100, N_{3}=50\right)$

\begin{tabular}{|cc||cccc||cc|ccc|}
\hline \multicolumn{1}{|l|}{} & \multicolumn{5}{c||}{$r=0.5$} & \multicolumn{3}{c|}{$r_{1}=0.5, r_{2}=0.3, r_{3}=0.2$} \\
\cline { 3 - 10 } & & Case 1 & Case 2 & Case 3 & Case 4 & Case 1 & Case 2 & Case 3 & Case 4 \\
\hline \hline$j=1$ & $M_{1 k}$ & 0.049 & 0.268 & 0.545 & 0.306 & 0.049 & 0.282 & 0.522 & 0.291 \\
& $A B_{1 k}$ & 0.049 & 0.229 & 0.455 & 0.260 & 0.049 & 0.241 & 0.435 & 0.248 \\
& $T_{1}$ & $\mathbf{0 . 0 4 9}$ & $\mathbf{0 . 3 3 6}$ & $\mathbf{0 . 5 1 1}$ & $\mathbf{0 . 3 8 5}$ & $\mathbf{0 . 0 4 8}$ & $\mathbf{0 . 3 5 4}$ & $\mathbf{0 . 4 8 6}$ & $\mathbf{0 . 3 6 7}$ \\
$j=2$ & $M_{2 k}$ & 0.051 & 0.062 & 0.647 & 0.385 & 0.047 & 0.054 & 0.645 & 0.377 \\
& $A B_{2 k}$ & 0.051 & 0.061 & 0.548 & 0.326 & 0.048 & 0.055 & 0.545 & 0.318 \\
& $T_{2}$ & $\mathbf{0 . 0 5 2}$ & $\mathbf{0 . 0 7 3}$ & $\mathbf{0 . 6 2 5}$ & $\mathbf{0 . 4 8 4}$ & $\mathbf{0 . 0 4 7}$ & $\mathbf{0 . 0 6 2}$ & $\mathbf{0 . 6 2 1}$ & $\mathbf{0 . 4 7 6}$ \\
$j=3$ & $M_{3 k}$ & 0.055 & 0.056 & 0.915 & 0.679 & 0.055 & 0.055 & 0.925 & 0.700 \\
& $A B_{3 k}$ & 0.055 & 0.055 & 0.843 & 0.589 & 0.054 & 0.055 & 0.856 & 0.609 \\
& $T_{3}$ & $\mathbf{0 . 0 6 0}$ & $\mathbf{0 . 0 6 2}$ & $\mathbf{0 . 9 1 9}$ & $\mathbf{0 . 7 9 3}$ & $\mathbf{0 . 0 6 0}$ & $\mathbf{0 . 0 6 2}$ & $\mathbf{0 . 9 3 0}$ & $\mathbf{0 . 8 1 4}$ \\
\hline
\end{tabular}

Table 8: Skew normal populations $\left(N_{1}=200, N_{2}=150, N_{3}=100\right)$

\begin{tabular}{|cc||cccc||cc|cc|}
\hline \multicolumn{1}{|c|}{} & \multicolumn{5}{c||}{$r=0.5$} & \multicolumn{3}{c|}{$r_{1}=0.5, r_{2}=0.3, r_{3}=0.2$} \\
\cline { 3 - 9 } & & Case 1 & Case 2 & Case 3 & Case 4 & Case 1 & Case 2 & Case 3 & Case 4 \\
\hline \hline$j=1$ & $M_{1 k}$ & 0.050 & 0.443 & 0.731 & 0.505 & 0.050 & 0.468 & 0.708 & 0.483 \\
& $A B_{1 k}$ & 0.050 & 0.369 & 0.628 & 0.422 & 0.050 & 0.390 & 0.605 & 0.403 \\
& $T_{1}$ & $\mathbf{0 . 0 4 9}$ & $\mathbf{0 . 5 5 2}$ & $\mathbf{0 . 7 8 1}$ & $\mathbf{0 . 6 2 4}$ & $\mathbf{0 . 0 4 9}$ & $\mathbf{0 . 5 8 2}$ & $\mathbf{0 . 7 5 7}$ & $\mathbf{0 . 5 9 9}$ \\
$j=2$ & $M_{2 k}$ & 0.050 & 0.068 & 0.814 & 0.594 & 0.049 & 0.061 & 0.816 & 0.593 \\
& $A B_{2 k}$ & 0.050 & 0.065 & 0.716 & 0.502 & 0.049 & 0.059 & 0.718 & 0.500 \\
& $T_{2}$ & $\mathbf{0 . 0 5 0}$ & $\mathbf{0 . 0 7 7}$ & $\mathbf{0 . 8 6 3}$ & $\mathbf{0 . 7 1 9}$ & $\mathbf{0 . 0 4 8}$ & $\mathbf{0 . 0 6 8}$ & $\mathbf{0 . 8 6 5}$ & $\mathbf{0 . 7 1 9}$ \\
$j=3$ & $M_{3 k}$ & 0.053 & 0.053 & 0.981 & 0.882 & 0.052 & 0.053 & 0.984 & 0.894 \\
& $A B_{3 k}$ & 0.052 & 0.053 & 0.947 & 0.802 & 0.052 & 0.053 & 0.953 & 0.812 \\
& $T_{3}$ & $\mathbf{0 . 0 5 5}$ & $\mathbf{0 . 0 5 5}$ & $\mathbf{0 . 9 9 2}$ & $\mathbf{0 . 9 5 2}$ & $\mathbf{0 . 0 5 4}$ & $\mathbf{0 . 0 5 5}$ & $\mathbf{0 . 9 9 3}$ & $\mathbf{0 . 9 5 9}$ \\
\hline
\end{tabular}

For the cases of unequal sample sizes, the simulation results reveal that all tests maintain the significance level for $j=1$ and 2 on the Case 1 . However, in Case 2 for $j=2$, the Mood test, Ansari-Bradley test and our test don't keep the significance level well. For $j=3$ in Case 1 and Case 2, the nonparametric tests maintain the significance level. Therefore the Mood test and Ansari-Bradley test are more robust than our test. In Case 3 of Table 7, the Mood test is more powerful than our test, but the power of our test is higher than the nonparametric statistics under other alternative hypothesis.

\section{Conclusion}

We develop a testing procedure for the statistic $T_{j}$ in a multipopulation and compare the power of the test using the statistic $T_{j}$ and two nonparametric tests. The limiting distribution of the proposed statistic is derived when the sample sizes are equal. In the case of unequal sample sizes, it is difficult to derive the limiting distribution of the $T_{j}$ statistic. Therefore we use the permutation test for the statistic $T_{j}$. In addition, we apply the testing procedure with the normal population, the contaminated normal population and the skew normal population and show that the estimated significance level of our test procedure, the Mood test and the Ansari-Bradley test converge for the significance level. Through the simulation studies, every test may be conservative for $j=1,2$ under the null hypothesis and the nonparametric tests are more robust than the test using the statistic $T_{j}$. However, the 


\section{MURAKAMI, TSUKADA and TAKEDA}

power of the test using the statistic $T_{j}$ is the greatest in both cases regardless of whether the sample sizes are equal or unequal. Therefore, we expect that the proposed test procedure is suitable for a multipopulation and this is shown by simulation.

\section{Acknowledgements}

The authors would like to thank the editor and the referees for valuable comments and suggestions. The authors are also grateful to use the high-performance computer systems in Department of Mathematics, Chuo University.

\section{REFERENCES}

Anderson, T. W. (2003). An Introduction to Multivariate Statistical Analysis, 3rd edition. New York: John Wiley.

Ansari, A. R. and Bradley, R. A. (1960). Rank sum tests for dispersion. Annals of Mathematical Statistics 31, 1174-1189.

Genton, M. G. (2004). Skew-elliptical Distributions and Their Applications. New York: Chapman \& Hall.

Gibbons, J. D. and Chakraborti, S. (2003). Nonparametric Statistical Inference, 4th edition. New York: Dekker.

Jackson, J. E. (2003). A User's Guide to Principal Components. New York: John Wiley.

Jolliffe, I. T. (2002). Principal Component Analysis, 2nd edition. New York: Springer.

Mood, A. M. (1954). On the asymptotic efficiency of certain nonparametric two-sample tests. Annals of Mathematical Statistics 25, 514-522.

Murakami, H., Hino, E. and Tsukada, S. (2007). Nonparametric test for eigenvalues of covariance matrix in multipopulation. Journal of the Japan Statistical Society 37, 299306.

Sugiyama, T. and Ushizawa, K. (1998). A non-parametric method to test equality of intermediate latent roots of two populations in a principal component analysis. Journal of the Japan Statistical Society 28, 227-235.

Takeda, Y. (2001). Permutation test for equality of each characteristic root in two populations. Journal of the Japanese Society of Computational Statistics 14, 1-10.

Tsai, W. S., Duran, B. S. and Lewis, T. O. (1975). Small-sample behavior of some multisample nonparametric tests for scale. Journal of the American Statistical Association $\mathbf{7 0}$, $791-796$.

(Received: December 3, 2007, Accepted: August 19, 2008) 